\title{
Programa para seleccionar y entrenar pacientes estandarizados en el contexto de un currículo universitario de simulación clínica
}

\author{
Roger Ruiz-Moral, Fernando Caballero-Martínez
}

Resumen. El artículo describe la metodología, estructura y organización para la selección y entrenamiento de pacientes estandarizados, en el contexto de un programa de pacientes estandarizados desarrollado en la Facultad de Medicina de la Universidad Francisco de Vitoria de Madrid. El objetivo del trabajo con pacientes estandarizados en este programa es la enseñanza, aprendizaje y evaluación de habilidades clínicas y comunicativas de los estudiantes de medicina.

Palabras clave. Educación médica. Grado. Habilidades clínicas. Habilidades comunicacionales. Métodos. Paciente simulado.

\section{A program for recruiting and training standardized patients in the context of a clinical simulation curriculum}

Summary. The paper describes the methodology, structure and organization for the recruitment and training of standardized patients in the context of a standardized patient program developed at the Francisco de Vitoria School of Medicine in Madrid. The goal of working with standardized patients in this program is to teach, learn and assess clinical and communication skills to medical students.

Key words. Clinical skills. Communication skills. Medical education. Methods. Simulated patient. Undergraduate.

\section{Introducción}

Un paciente estandarizado (PE) es un individuo al que se entrena para representar de forma coherente y precisa, generalmente ante estudiantes, médicos o enfermeros, una enfermedad o problema de salud concreto con el objetivo de enseñar y evaluar habilidades interpersonales y clínicas. La evidencia ha demostrado que los PE constituyen un método de enseñanza eficaz que proporciona a los estudiantes una experiencia de aprendizaje auténtica en una determinada materia y en contextos educativos y prácticos concretos [1]. En líneas generales, al PE se le entrena y capacita para representar ciertos roles con determinadas características del lenguaje corporal y para que responda a las preguntas que le hacen los entrevistadores de forma adecuada [2,3]. La autenticidad y el feedback son las dos variables principales que determinan la calidad de la actuación y el entrenamiento de un PE [4]. El entrenamiento dado al PE es la mejor forma de garantizar la autenticidad y reproducibilidad de un encuentro clínico con un PE [4-6].

Este artículo pretende describir los pasos necesarios para llevar a cabo el proceso de captación y for- mación de un PE con el objetivo de entrenar y evaluar a los estudiantes de medicina en habilidades comunicativas de una manera coherente y estandarizada. El conjunto de estas recomendaciones conforman el Programa de Entrenamiento de Pacientes Estandarizados de la Facultad de Medicina de la Universidad Francisco de Vitoria de Madrid (PPE_UFV).

\section{Selección de los pacientes estandarizados}

Casi cualquier persona puede ser entrenada para actuar como PE, incluyendo a personal sanitario, si tiene interés, capacidad y motivación suficientes, y si le gusta el tema y el hecho de actuar. Es probable que las opiniones y actitudes negativas previas hacia los sanitarios o los profesionales médicos sea un impedimento mayor para admitir a un aspirante al programa [5]. No tienen por qué ser actores profesionales o aficionados (éstos tal vez tiendan a sobreactuar) [5-7]. Aunque hay que tener en cuenta muchos factores a la hora de seleccionar a un posible PE, lo ideal es que su perfil se ajuste todo lo posible al caso o rol que estemos preparando. Por ejemplo, si pensamos en un caso que evalúe las ha-
Director del Programa de Comunicación Clínica (R. Ruiz-Moral). Director Académico de la Facultad de Medicina (F. Caballero-Martínez). Universidad Francisco de Vitoria. Madrid, España.

Correspondencia:

Prof. Roger Ruiz Moral. Facultad de Medicina. Universidad Francisco de Vitoria. Ctra. M-515, PozueloMajadahonda, km 1,800. E-28223 Pozuelo de Alarcón (Madrid).

E-mail:

r.ruiz.prof@ufv.es

Conflicto de intereses: No declarado.

Competing interests: None declared.

(c) 2014 FEM 
bilidades para motivar a una paciente obesa y de mediana edad a seguir una dieta y ejercicio, $u$ otro cuyo objetivo sea obtener información en la entrevista con una paciente adolescente en la que se sospecha un trastorno alimentario como la anorexia, los perfiles demográficos y fenotípicos requeridos serán obviamente muy distintos. A veces esto no es fácil de conseguir y la dificultad para encontrar al candidato idóneo (especialmente si estamos presionados por límites de tiempo o costes) puede inducirnos a modificar el caso hacia otro perfil no tan adecuado para los objetivos planteados. Esta decisión puede tener repercusiones negativas en la enseñanza, por lo que conviene prevenirlas disponiendo antes de un grupo de personas amplio, diverso y comprometido con estos fines.

Con estos supuestos se creó el PPE_UFV. Como primer paso, el programa incorporó una estrategia de selección que abarcó anuncios para colectivos concretos como centros cívicos o grupos de teatro aficionado pertenecientes a la zona geográfica y a los propios miembros de la comunidad universitaria. Como segundo paso, durante un período de cinco meses se envió a las personas interesadas una información básica sobre el PPE_UFV así como un dossier que el interesado debía cumplimentar y que contenía distinta información personal de interés para una adecuada evaluación del candidato [7]. Como tercer paso, a todos aquellos que contestaron enviando su dossier personal, se les citaba para una sesión colectiva con el objetivo de un primer contacto y una explicación presencial del programa, así como para aclarar dudas al respecto. Esta primera reunión (que se convocó en cuatro ocasiones) incluía la descripción de los objetivos del programa, su planteamiento general, los compromisos que asume un $\mathrm{PE}$, los planes inmediatos (a un año vista) previstos en cuanto a número necesario y tipos de escenarios, y los aspectos económicos más relevantes. La retribución económica varía dependiendo de la carga que suponga el tipo de actividad a realizar y, aunque no hay un criterio uniforme, suele oscilar entre los 15-30 euros por hora de dedicación [8]. De esta forma, en el momento presente, el PPE_UFV cuenta con un total de 32 personas incorporadas y disponibles, con un perfil demográfico (y clínico) variado y con un rango de edades de 16-78 años.

\section{Entrenamiento de los pacientes estandarizados: generalidades}

El entrenamiento de un PE varía en función del tipo de rol (sus objetivos docentes) y del escenario asig- nado. En nuestro programa, los PE participan asumiendo roles completos de pacientes con objetivos variados (comunicativos o de habilidades clínicas) para interacciones docentes y evaluativas con estudiantes (con o sinfeedback), en seminarios, talleres, clases o evaluaciones clínicas objetivas y estructuradas (ECOE), y también para interpretar situaciones clínicas específicas (escenario de confidencialidad, prevención, obtener determinado tipo de información delicada...), que son grabadas en video y que, como material docente ('videos gatillo'), pasan a formar parte de un repositorio de situaciones y casos para la enseñanza en talleres y seminarios.

Tradicionalmente, y para la educación médica, este tipo de entrenamiento puede requerir de 4 a 20 horas, con un número variable de sesiones [3,5-7]. Todo esto depende de si en el desempeño del PE se incluye dar o no feedback a los estudiantes, de la complejidad del caso y de lo experimentado que sea el PE. Sin embargo, con independencia de esto, ante todo nuevo caso y evento, cualquier PE debe pasar por una fase específica de entrenamiento. Este entrenamiento puede desarrollarlo personal médico o sanitario de otro tipo (dependiendo del caso), aunque si los escenarios incluyen signos, síntomas o determinadas conductas de enfermedad, se requiere la supervisión sistemática de un clínico [3,5].

\section{Entrenamiento de los pacientes estandarizados en el PPE_UFV}

Se describe aquí el entrenamiento que siguen los $\mathrm{PE}$ sin experiencia previa (nuevos) para interpretar un rol completo ante estudiantes ofreciéndoles feedback, dado que es la modalidad más habitual y tal vez la más completa.

Este entrenamiento incluye una sesión preliminar introductoria, dos sesiones principales o talleres de entrenamiento específico, y alguna o algunas sesiones complementarias más breves y con objetivos más limitados. Una vez diseñado el caso o casos necesarios y elegidas las fechas del evento educativo a desarrollar (considerado éste como el conjunto de días y horas concretas planificados de la facultad para las actividades docentes con los estudiantes), se procede a elegir del pool universitario de PE aquellos que a priori mejor se adaptan a cada uno de los roles y se les cita para la sesión preliminar.

\section{Sesión preliminar}

El propósito de esta sesión es, sobre todo, explicativo. En ella se ofrecen detalles del caso clínico, se ex- 
plica la organización del entrenamiento (incluyendo las fechas de los talleres presenciales), se fijan las condiciones y fechas previstas para el evento, y se concretan las condiciones económicas contempladas. Se aprovecha también esta sesión para clarificar los asuntos relativos a la confidencialidad sobre el caso y para obtener la firma de los PE en un documento por el que se comprometen a no compartir el caso con nadie más. De igual forma, los PE firman entonces un consentimiento para ser videograbados y para que sus intervenciones puedan utilizarse con fines docentes en la universidad, así como un compromiso de cumplimiento por su parte de las normas establecidas. En esta reunión se les facilita una documentación inicial consistente en la descripción escrita de todos los detalles del caso, un manual de entrenamiento y feedback, una agenda temporal con las fechas del evento y de los entrenamientos requeridos, y un cuaderno de bitácora donde podrán registrar el número de horas que dedicarán a todo el proceso, con fines contables para la gestión del cobro de honorarios.

La tarea inmediata que se pide a cada alumno-PE (A-PE) es estudiar a fondo todos los detalles del caso, para lo que se facilita una comunicación electrónica/telefónica permanente con el monitor. Posteriormente (4-7 días después) se le envía un CD con tres videos y otras tantas interacciones PE-estudiantes, todas sobre un mismo caso y con diferentes niveles de calidad (mala, aceptable y buena), así como los objetivos y los detalles principales de dicho caso que le serán necesarios para poder evaluar en los videos el papel del PE. Los A-PE deben realizar esta evaluación utilizando una lista de comprobación, el 'listado evaluativo de la calidad de la actuación del PE' (LE_PE), que tiene un formato de checklist de 15 ítems sobre aspectos claves de la actuación del PE (Tabla I).

Una vez que los PE se comprometen para el evento concreto con los roles asignados, se contacta con otros tantos PE previamente entrenados en casos con objetivos similares y se les ofrece su participación en el evento como 'suplentes'. Generalmente, ante un evento que requiere más de un caso y de un $\mathrm{PE}$, el entrenamiento se hace conjuntamente con todos los PE implicados (salvo los suplentes).

\section{Primer taller formativo}

Entre 7-10 días después del primer contacto, se cita a los A-PE a una segunda sesión o primer taller formativo. Se espera que, hasta esa fecha, los A-PE hayan trabajado las tareas anteriormente asignadas. La duración de este taller, variable en función del
Tabla I. Listado evaluativo de la calidad de la actuación del paciente estandarizado a.

El paciente de esta entrevista parece real (el rol que interpreta es creíble)

Su proxémica y resto del lenguaje no verbal son adecuados

Se comporta de manera natural a lo largo de toda la entrevista (no sobreactúa ni se muestra coartado)

Tiene coherencia emocional (la expresión de las emociones es adecuada)

Tiene recursos para el diálogo (es capaz de hablar sobre los temas que van saliendo sin regalar pistas)

Responde adecuadamente a las preguntas del entrevistador (conoce bien su rol)

Claves obligadas: las expone todas

Claves obligadas: las expone en los momentos adecuados

Claves de seguimiento: las expone tras preguntas o comportamientos del entrevistador que lo requieren

Claves de seguimiento: ocultó alguna/s (que debió exponer)

Claves de seguimiento: regaló alguna/s (que no debió exponer)

No interrumpe al entrevistador

Responde a todas las preguntas del entrevistador

Improvisa adecuadamente

Valoración global del alumno

a Los 15 ítems se evalúan como 'bueno', 'promedio' o 'debe mejorar'.

número de A-PE y casos a entrenar, suele ser de 4-5 horas para tres alumnos y casos. Los objetivos de este primer taller son los siguientes:

- Revisión general del proceso de aprendizaje y de los aspectos generales y comunes a los tres casos (comentarios y dudas). Repaso de todos los aspectos relativos a cada caso específico y comentarios sobre dudas que puedan tener, especialmente sobre su puesta en escena.

- Revisión de los ítems del LE_PE y del trabajo de cada alumno con los videos. La revisión que han hecho los A-PE de los vídeos les supone una primera aproximación a diferentes estilos de actuación de un PE y también de los entrevistadores. El uso del LE_PE les ayuda tomar conciencia de los aspectos importantes que deben tener en cuenta cuando interpreten el rol y les sirve de orientación inicial para dar sentido a la entrevista. En esta primera etapa no se les exige un estricto rigor evaluativo, y su utilidad radica en visualizar distintos tipos de interacciones y actuaciones y servir de base para la discusión. 
Tabla II. Puntos clave del entrenamiento con pacientes simulados.

Detallar y especificar algunos síntomas/signos, características físicas, expresiones faciales requeridas

Insistir en la necesidad de que el paciente estandarizado interiorice

y 'sienta' como si en realidad fuese la persona/paciente a la que interpreta

Insistir en la importancia de que, ante actitudes o comportamientos similares, todos los encuentros deberían ser consistentes de un estudiante a otro

Estar abiertos y animar a los pacientes estandarizados a que pregunten

lo que no entiendan y asegurarse de que comprenden lo que se les dice

Acomodarse a las preguntas que los pacientes estandarizados hagan, no desacreditarlos

Si los estudiantes hacen preguntas o realizan habilidades que no se han tratado en los entrenamientos el paciente estandarizado debería mostrarse todo lo auténtico que pueda y responder con el enfoque que mejor se adapte al caso ción postencuentro de éste (FON_PE). Este listado varía en función de los objetivos de la entrevista. El hecho de que diversos A-PE hagan conjuntamente este taller y el resto del proceso formativo es especialmente útil porque les da la oportunidad de ir observando e interaccionando unos con otros, lo que enriquece mucho su formación y ahorra tiempo al monitor.

Tras este taller, los A-PE deben revisar las videograbaciones de sus entrevistas con el monitor utilizando el LE_PE. Con ello afianzan el conocimiento del caso, una vez que ya controlan los aspectos más prácticos y básicos de su implementación.

\section{Segundo taller formativo}

Con el fin de consolidar el programa de entrenamiento se desarrolla un segundo taller que tiene por objetivos principales el afianzamiento del rol y su interpretación, y el entrenamiento en la evaluación de la actuación del estudiante mediante el uso del FON_PE. Para ello se sigue una estructura similar al primer taller, iniciándose con una revisión general del proceso de aprendizaje y de los aspectos generales y comunes a los tres casos. Aquí se incluye una discusión sobre sus impresiones una vez han visto sus propias entrevistas y las han evaluado. Se les pide que hagan una crítica constructiva (al estilo del feedback que a la vez están aprendiendo) sobre la evaluación que han hecho de sí mismos: detección de sus fortalezas y áreas de mejora. Tras ello, el monitor pasa a interpretar con cada A-PE dos o tres interacciones más de su rol, mostrando, una vez más, diferentes modelos de entrevistador. Estas entrevistas vuelven a ser videograbadas. Al finalizar cada entrevista, el A-PE debe ofrecer feedback al monitor sobre su actuación y evaluarla con el listado de comprobación FON_PE. Tras esto, el monitor ofrece feedback sobre la actuación del PE y sobre el feedback que éste ha realizado. Para completar el ciclo de aprendizaje de las habilidades exigidas se pide a los A-PE que, en su domicilio, revisen las últimas grabaciones y se vuelvan a evaluar ellos mismos usando el LE_PE.

\section{Fase final del entrenamiento}

Esta fase tiene ya lugar en escenarios 'reales', es decir, cuando el A-PE se enfrenta a interacciones con estudiantes dentro del programa docente de éstos. Los dos escenarios habituales usados con tal fin son seminarios en grupo y encuentros individualizados con estudiantes, incluidos en nuestro programa edu- 
cativo. En general, antes de estos encuentros se suele mantener una sesión recopilatoria breve (unos 30 minutos de recordatorio de los aspectos claves del rol que el PE debe interpretar).

- Seminarios. En los seminarios, de dos horas de duración, cuatro alumnos tienen otras tantas interacciones consecutivas con el PE. Tras cada una de estas interacciones, efectuadas en una cámara Gesell (una consulta acondicionada para permitir la observación directa desde un aula, a través un vidrio de visión unilateral, con equipos de audio y videograbación), que son visualizadas por el resto de los 14-16 estudiantes participantes y el monitor, el PE y el estudiante pasan al aula, donde el primero ofrece feedback directo. Tras finalizar el seminario, el monitor y el A-PE mantienen una sesión donde abordan los aspectos más relevantes de la actuación y el feedback ofrecido por aquél.

- Encuentros individualizados. Todos los encuentros individualizados son videograbados y archivados utilizando una plataforma tecnológica de gestión documental y grafica (LearningSpace ${ }^{\circledR}$ ), una herramienta informática que automatiza el registro y clasificación de todo el material producido en cualquiera de los espacios del Centro de Simulación (consultas, planta de hospitalización, boxes de urgencias, domicilio, UVI móvil...). Este instrumento permite a cualquier participante recuperar en diferido y vía internet sus grabaciones y realizar evaluaciones de éstas. Generalmente, las interacciones duran diez minutos y el PE tiene cinco minutos adicionales antes de la siguiente interacción, momento en el que debe realizar la evaluación del estudiante. El profesor evalúa cada uno de los encuentros seleccionando y anotando los aspectos más relevantes de algunas de las actuaciones del PE, para abordarlos conjuntamente en una última sesión de debriefing.

En la tabla II se detallan los puntos clave a abordar en el conjunto de sesiones con los A-PE. La figura ofrece una visión general de los pasos y contenidos del proceso de entrenamiento descrito.

\section{Conclusiones}

Aunque varias facultades e instituciones en España trabajan desde hace tiempo utilizando metodologías docentes relacionadas con la exposición a escenarios simulados mediante dinámicas de role-play, para la enseñanza de habilidades clínicas y comunicativas, hasta donde conocemos, no existe en nues-
Figura. Esquema del proceso del primer entrenamiento a un paciente estandarizado nuevo en el PPE_UFV.

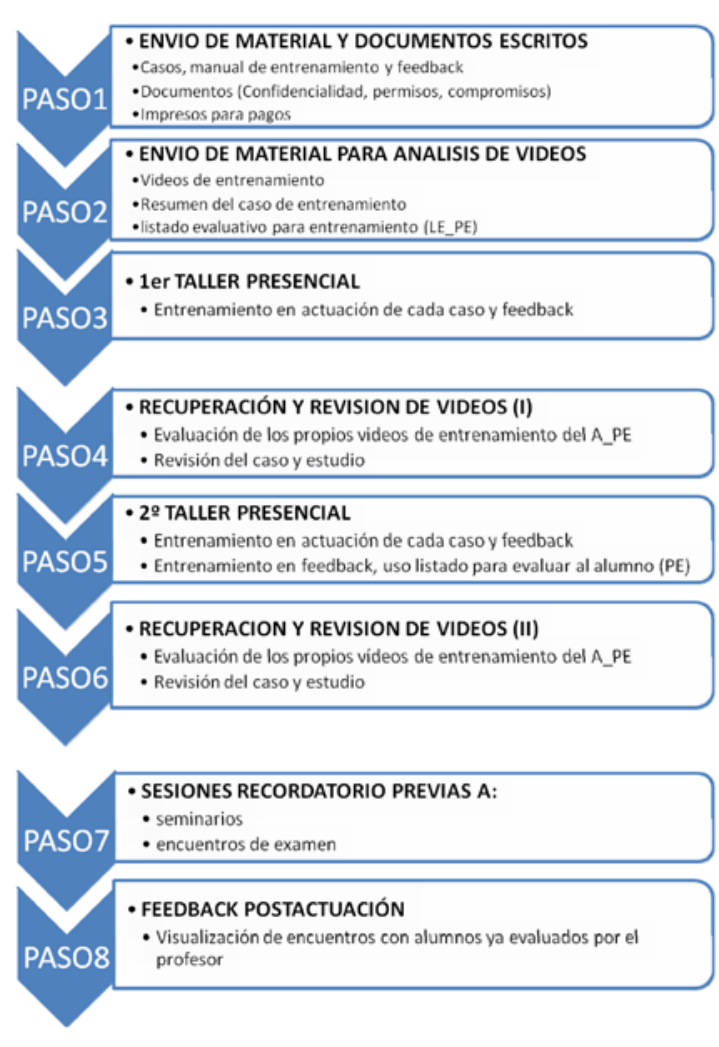

tro país una experiencia similar que haya desarrollado un programa completo de las características del aquí presentado. Tras dos años de recorrido, el PPE_UFV ha demostrado ser viable y responder a los fines requeridos, especialmente para la selección y el entrenamiento de PE. Disponer de este recurso ha repercutido directamente en los resultados de aprendizaje de nuestros estudiantes, en cuanto a la adquisición de habilidades comunicativas constatables en las ECOE desarrolladas al final de tercer y cuarto cursos.

Aunque aún no disponemos de datos que muestren en qué medida los estudiantes han progresado por encima de otros programas o estrategias docentes en la adquisición de este tipo de habilidades, el hecho de que algo más de un 50\% de ellos hayan superado los objetivos de aprendizaje preestablecidos después de un programa predeterminado (compuesto de tres seminarios grupales y dos encuentros individuales con un PE) y que un $90 \%$ superaran con éxito la evaluación de las dos asignaturas 
que recogen este tipo de habilidades prácticas (donde representan el $65 \%$ de la nota final), sugiere que el programa y el sistema de entrenamiento expuestos son válidos. Sin embargo, será preciso desarrollar paralelamente una agenda de investigación en la evaluación de otros aspectos imprescindibles de este programa, como son la fiabilidad de las actuaciones de los PE en diferentes contextos, el rigor de sus evaluaciones y la correlación de éstas con las evaluaciones realizadas por profesores, entre otros muchos aspectos.

Es evidente que en el proceso de uso docente del $\mathrm{PE}$ en la formación pregraduada existen otros factores determinantes del éxito que deben garantizarse (criterio técnico y clínico suficientes para diseño de casos, selección de los objetivos docentes adecuada al perfil de los destinatarios de la formación, validez y fiabilidad de sistemas de evaluación de las actuaciones, recursos humanos accesibles y entrenados, viabilidad presupuestaria del programa, acceso a un dispositivo docente adaptado a este tipo de formación, etc.). Aun así, la posible cobertura de objetivos complementarios (formación continuada, posgrado...) y las amplias posibilidades que ofrece un programa de esta naturaleza justifican el desa- rrollo creciente de este tipo de actividades docentes, consideradas, en otro países, imprescindibles para la correcta enseñanza y evaluación de las competencias clínicas y comunicativas en estudiantes de medicina y otras carreras sanitarias afines.

\section{Bibliografía}

1. Barrows HS. An overview of the uses of standardized patients for teaching and evaluating clinical skills. Acad Med 1993; 68: 443-5.

2. Stillman PL, Regan MB, Philbin M, Haley M. Results of a survey on the use of standardized patients to teach and evaluate clinical skills. Acad Med 1990; 65: 288-92.

3. Stillman PL. Technical issues: logistics. Acad Med 1993; 68: 464-70

4. Wind LA, Dalen JV, Arno MMM, Rethans JJ. Assessing simulated patients in an educational setting: the MASP (Maastricht Assessment of Simulated Patients). Med Educ 2004; 38: 39-44.

5. Chur-Hansen A, Burg F. Working with standardised patients for teaching and learning. Clin Teach 2006; 3: 220-4.

6. Ruiz-Moral R, Zarco-Montejo J. Enseñanza con pacientes simulados. In Ruiz-Moral R, ed. Educación médica, manual práctico para clínicos. Madrid: Editorial Médica Panamericana; 2010. p. 187-90.

7. Cleland JA, Abe K, Rethans JJ. The use of simulated patients in medical education: AMEE Guide No. 42. Med Teach 2009; 39: 477-86.

8. Howley LD, Gliva-McConvey G, Thornton J. Standardized patient practices: initial report on the survey of U.S. and Canadian medical schools. Med Educ Online 2009; 14: 1-7. 